\title{
Mitochondrial genome of the nonphotosynthetic mycoheterotrophic plant Hypopitys monotropa, its structure, gene expression and RNA editing
}

\author{
Viktoria Yu Shtratnikova ${ }^{1}$, Mikhail I Schelkunov $^{2}$, Aleksey Penin $^{3}$, Maria D Logacheva ${ }^{\text {Corresp. } 2}$ \\ ${ }^{1}$ A. N. Belozersky Institute of Physico-Chemical Biology, Lomonosov Moscow State University, Moscow, Russia \\ 2 Skolkovo Institute of Science and Technology, Moscow, Russia \\ 3 Laboratory of Plant Genomics, Institute for Information Transmission Problems of the Russian Academy of Sciences, Moscow, Russia \\ Corresponding Author: Maria D Logacheva \\ Email address: maria.log@gmail.com
}

Heterotrophic plants-plants that have lost the ability to photosynthesize-are characterized by a number of changes at all levels of organization. Heterotrophic plants are divided into two large categories-parasitic and mycoheterotrophic. The question of to what extent such changes are similar in these two categories is still open. The plastid genomes of nonphotosynthetic plants are well characterized, and they exhibit similar patterns of reduction in the two groups. In contrast, little is known about the mitochondrial genomes of mycoheterotrophic plants. We report the structure of the mitochondrial genome of Hypopitys monotropa, a mycoheterotrophic member of Ericaceae, and the expression of its mitochondrial genes. In contrast to its highly reduced plastid genome, the mitochondrial genome of $H$. monotropa is larger than that of its photosynthetic relative Vaccinium macrocarpon, and its complete size is $\sim 810 \mathrm{Kbp}$. We observed an unusually long repeat-rich structure of the genome that suggests the existence of linear fragments. Despite this unique feature, the gene content of the $H$. monotropa mitogenome is typical of flowering plants. No acceleration of substitution rates is observed in mitochondrial genes, in contrast to previous observations in parasitic non-photosynthetic plants. Transcriptome sequencing revealed the trans-splicing of several genes and RNA editing in 33 of 38 genes. Notably, we did not find any traces of horizontal gene transfer from fungi, in contrast to plant parasites, which extensively integrate genetic material from their hosts. 
1 Mitochondrial genome of the nonphotosynthetic mycoheterotrophic plant Hypopitys

2 monotropa, its structure, gene expression and RNA editing

Email address: maria.log@gmail.com

\author{
Viktoria Y. Shtratnikova ${ }^{1}$, Mikhail I. Schelkunov ${ }^{2,3}$, Aleksey A. Penin ${ }^{2}$, Maria D. Logacheva ${ }^{1,2,3}$
}

${ }^{1}$ A. N. Belozersky Institute of Physico-Chemical Biology, Lomonosov Moscow State University, Moscow, Russian Federation

${ }^{2}$ Laboratory of Plant Genomics, Institute for Information Transmission Problems of the Russian

Academy of Sciences, Moscow, Russian Federation

${ }^{3}$ Skolkovo Institute of Science and Technology, Moscow, Russian Federation

Corresponding Author:

Maria D. Logacheva

16

\title{
Abstract
}

Heterotrophic plants - plants that have lost the ability to photosynthesize - are characterized by a number of changes at all levels of organization. Heterotrophic plants are divided into two large categories - parasitic and mycoheterotrophic. The question of to what extent such changes are similar in these two categories is still open. The plastid genomes of nonphotosynthetic plants are well characterized, and they exhibit similar patterns of reduction in the two groups. In contrast, little is known about the mitochondrial genomes of mycoheterotrophic plants. We report the structure of the mitochondrial genome of Hypopitys monotropa, a mycoheterotrophic member of Ericaceae, and the expression of its mitochondrial genes. In contrast to its highly reduced plastid genome, the mitochondrial genome of H. monotropa is larger than that of its photosynthetic relative Vaccinium macrocarpon, and its complete size is $\sim 810 \mathrm{Kbp}$. We observed an unusually long repeat-rich structure of the genome that suggests the existence of linear fragments. Despite this unique feature, the gene content of the H. monotropa mitogenome is typical of flowering plants. No acceleration of substitution rates is observed in mitochondrial genes, in contrast to previous observations in parasitic non-photosynthetic plants. Transcriptome sequencing revealed the trans-splicing of several genes and RNA editing in 33 of 38 genes. Notably, we did not find any traces of horizontal gene transfer from fungi, in contrast to plant parasites, which extensively integrate genetic material from their hosts.

\section{Introduction}

Hypopitys monotropa (Ericacae, Monotropoideae) is a nonphotosynthetic plant that obtains carbon from fungi in ectomycorrhizal relationships with tree roots (Bjorkman, 1960). In contrast to most other mycoheterotrophic (MHT) plants, which are very rare and/or very narrowly distributed, Monotropoideae, including H. monotropa, are quite widespread and are associated with old-growth conifer forests. Thus, H. monotropa is used as a model system in studies of 
41 plant-mycorrhizal associations and the developmental biology of MHT plants (e.g., Olson, 1993, 1990). Recent advances in DNA sequencing have expanded research on mycoheterotrophs into genomics. To date, most attention has been focused on the plastid genomes of MHT plants, which are highly reduced in size and gene content, including a complete absence or pseudogenization of genes of the photosynthesis electron transport chain (for a review, see Graham et al., 2017). Thus, the MHT lifestyle strongly affects plastids, but what about the mitochondrial genome?

In contrast to animals, in which mitochondrial genomes are usually conserved in size and gene content across large taxonomic groups, in plants, these genomes are highly variable and may be very dissimilar even in closely related species. The size of the angiosperm mitogenome ranges from $66 \mathrm{~Kb}$ in the hemiparasitic mistletoe Viscum scurruloideum (Skippington et al., 2015) and $222 \mathrm{~Kb}$ in the autotrophic Brassica napus (Handa, 2003) to more than $11 \mathrm{Mb}$ in Silene noctiflora. Despite such huge variations in size, a large fraction of mitochondrial genes - those that encode the components of the oxidative phosphorylation chain complexes and proteins involved in the biogenesis of these complexes-are stable in content and show very low sequence divergence. More variation exists in the group of genes involved in translation, i.e., ribosomal proteins and transfer RNAs (Adams et al., 2002; Gualberto et al., 2014), presumably due to the transfer to the nuclear genome that occurred in several plant lineages or the nonessentiality of these genes. In addition, many plant mitochondrial genomes carry open reading frames (ORFs) that potentially encode functional proteins (Qiu et al., 2014); such ORFs are highly lineage specific. Nonphotosynthetic plants are divided into two large groups - those that are parasitic on other plants and those that are mycoheterotropic. To date, only a few complete mitochondrial genomes of nonphotosynthetic plants have been characterized, and most of them belong to parasitic species. A comparative analysis of the mitogenomes of several parasitic, hemiparasitic and autotrophic Orobanchaceae (Fan et al., 2016) showed that the gene content does not depend on trophic specialization in the family range. The mitogenomes of two nonrelated lineages of parasitic plants, Rafflesiaceae and Cynomoriaceae, also do not show a reduction in gene content, and they provide an example of massive HGT from other plants (including but not limited to their hosts). This is not, however, a trait that is unique to parasitic plants-e.g., in Amborella trichopoda, an autotrophic plant from the basal angiosperms, includes a large fraction of the mitochondrial genome was acquired from green algae, mosses, and other angiosperms (Rice et al., 2013). In contrast, in the hemiparasitic plant Viscum scurruloideum, the mitogenome is drastically reduced in size and gene content, and it lacks all nine nad genes and matR (Skippington et al., 2015). The mitogenomes of other Viscum species are not reduced in length but exhibit a reduced gene content, similar to that of $V$. scurruloideum ((Petersen et al., 2015); but see (Skippington et al., 2017)). The available sampling is obviously insufficient even for parasitic plants; regarding mycoheterotrophs, the data are almost completely lacking. Only two mitochondrial genomes of MHT plants have been characterized by date - those of the orchid Gastrodia elata (Yuan et al., 2018) and Epirixanthes elongata (Polygalaceae) (Petersen et al., 2019). The former was characterized as a part of a genome sequencing project and is not 
81 completely assembled, being represented by 19 contigs. The $G$. elata mitogenome is large $(\sim 1.3$ $82 \mathrm{Mb})$, while that of E. elongata is much smaller $(\sim 0,4 \mathrm{Mb})$. However, both of these studies lack a 83 comparative analysis with autotrophic relatives that would allow us to highlight the changes 84 associated with mycoheterotrophy.

85 In this context, we set the following objectives: 1) to characterize the structure and gene content 86 of the mitochondrial genome of H. monotropa, 2) to estimate the possibility of horizontal gene transfer (HGT) from fungi, and 3) to study mitochondrial gene expression and RNA editing. The photosynthetic plant with a characterized mitochondrial genome that is phylogenetically closest to H. monotropa is cranberry, Vaccinium macrocarpon (Fajardo et al., 2014). In this study, we used $V$. macrocarpon as a basis for comparative analysis aimed at identifying the patterns (if any) of mitochondrial genome changes associated with mycoheterotrophy.

92 Materials \& Methods

\subsection{Sample collection and sequencing}

The procedures for ample collection, DNA and RNA library preparation for most datasets (DNA shotgun and mate-pair libraries and an inflorescence transcriptome library) and sequencing were described in the reports of Logacheva et al. (2016) and Schelkunov et al. (2018). In addition to the data generated in previous studies, we used four new datasets that represent the transcriptomes of the anthers and ovules of H. monotropa. The samples were collected in the same location as previous samples but in 2018. Hypopitys monotropa is not an endangered or threatened plant species; thus, no specific permissions were required for its collection. RNA was extracted using an RNEasy kit (Qiagen) with the addition of the Plant RNA Isolation Aid reagent (Thermo Fisher) to the lysis buffer. The removal of ribosomal RNA was performed using a RiboZero plant leaf kit (Illumina), and further sample preparation was performed using a NEBNext RNA library preparation kit (New England Biolabs). The libraries were sequenced on the HiSeq 4000 platform (Illumina) in 150-nt paired-end mode. The reads were deposited in the NCBI Sequence Read Archive under BioProject PRJNA573526.

107 2.2. Assembly

108 Read trimming and assembly were performed as described in the report of Logacheva et al. 109 (2016). The contig coverage was determined by mapping all reads in CLC Genomics Workbench

110 v. 7.5 .1 (https://www.qiagenbioinformatics.com/products/clc-genomics-workbench/), requiring 111 at least $80 \%$ of a read's length to align with at least $98 \%$ sequence similarity. To find contigs

112 corresponding to the mitochondrial genome, we performed BLASTN and TBLASTX alignment 113 of the Vaccinium macrocarpon genome (GenBank accession NC_023338) against all contigs in 114 the assembly. BLASTN and TBLASTX from the BLAST 2.3.0+ suite (Camacho et al., 2009) 115 were used, and the alignment was performed with a maximum allowed $e$-value of $10^{-5}$. Low116 complexity sequence filters were switched off to avoid missing genes with an extremely high or 117 low GC content. A contig that corresponded to the plastid genome of Hypopitys monotropa also 118 aligned to the mitochondrial genome of Vaccinium macrocarpon because of the presence of 119 inserts from the plastid genome in the mitochondrial genome. The complete sequence of the $H$. 120 monotropa plastome is known from our previous study (Logacheva et al., 2016) and was 
121 excluded from further analyses. Contigs showing coverage of less than 10 or those that exhibited

122 matches only to non-mitochondrial sequences when aligned to the NCBI nt database by

$123 \operatorname{BLASTN}\left(e\right.$-value $10^{-3}$ ) were considered nuclear.

124 After the procedures described above, 7 contigs remained, with lengths of 255, 5651, 20627 ,

$125102868,106298,234240$, and $309413 \mathrm{bp}$ and coverages of 37, 89, 93, 110, 96, 107, and $108 \mathrm{bp}$,

126 respectively. The lower coverage of the smallest contig was presumably an artefact caused by its

127 length. To understand their order in the mitochondrial genome, we mapped the mate-pair reads

128 from the library with the largest insert size $(8705 \pm 2537 \mathrm{bp})$ to the contigs and investigated the

129 mate-pair links. The mapping was performed by using CLC Genomics Workbench, and 100\% of

130 a read's length was required to align to a contig with a sequence similarity of $100 \%$, to minimize

131 the amount of incorrectly mapped reads. Mate-pair links were visualized by using Circos 0.67-5

132 (Krzywinski et al., 2009) and investigated manually. In one contig, the mate-pair links spanned

133 the entire length from the end to the start; thus, the contig corresponded to a circular

134 chromosome. The other six contigs were connected into a linear sequence, whose structure is

135 described in the Results section. Gaps between the contigs were closed by GapFiller (Boetzer

136 and Pirovano, 2012), which was run with the following parameters: -m 30 -o 2 -r 0.8 -n 50 -d

137100000 -t 100 -g 0 -T 4 -i 100000. The source code of GapFiller was rewritten to allow it to use

138 Bowtie 2 as the read mapper. Additionally, we tried to extend the edges of the linear chromosome

139 by using GapFiller to check that there were no assembly forks (sites with two alternative

140 extensions). The absence of such forks indicates that the linearity of the sequence is not a

141 consequence of an assembly problem. Instead, during extension, the coverage gradually

142 decreased to zero, implying a linear chromosome with lengths varying between different copies

143 of the chromosome. To check whether the two chromosomes were assembled correctly, we

144 mapped the reads from all three sequencing libraries by using CLC Genomics Workbench v.

1457.5 .1 , requiring at least $90 \%$ of a read's length to align with at least 99\% sequence similarity and

146 checked that the coverage was approximately uniform along the whole length of the

147 chromosomes. As observed during the analysis by GapFiller, we found that the coverage

148 gradually decreased at the edges of the linear chromosome, indicating that different copies of the

149 chromosome in a plant exhibit different lengths of the terminal regions. For the sake of precision,

150 we elongated the terminal regions such that the coverage dropped to 0 ; thus, the length of the

151 linear chromosome approximately corresponded to the maximal possible length among all copies

152 of the chromosome in the sequenced plant. Additionally, we mapped the reads from the mate-

153 pair library with the largest insert sizes, requiring $100 \%$ of a read's length to align with a

154 sequence similarity of $100 \%$, and we investigated the distribution of mean insert sizes over each

155 genomic position and the number of mate pairs than spanned each position. These values were

156 approximately uniform along both chromosomes (Supplementary Figure S1), suggesting that the

157 assembly was correct.

158 2.3. Mitogenome annotation

159 Initial annotation was performed using Mitofy (Alverson et al., 2010) with further manual 160 correction. To verify exon boundaries, we mapped RNA-seq reads to the sequences of the 
161 chromosomes by using CLC Genomics Workbench v. 7.5.1, requiring at least 80\% of a read's

162 length to map with at least $90 \%$ sequence similarity. The relaxed setting for the percentage of

163 read length to be mapped allows the mapping reads to span splice junctions, and the relaxed

164 setting for sequence similarity allows the mapping of reads to regions with a high density of

165 RNA editing sites. The alignment was visualized in CLC Genomics Workbench 7.5.1.

166 To check if there were any protein-coding genes that were missed, we visually inspected the

167 alignment to search for unannotated regions in the chromosomes with high coverage by RNA-

168 seq reads. This search did not add new genes.

169 The search for genes encoding selenocysteine tRNAs (tRNA-Sec) was performed for Hypopitys

170 monotropa and Vaccinium macrocarpon by using an online version of Secmarker 0.4

171 (Santesmasses et al., 2017) with the default parameters. SECIS element prediction was

172 performed by using an online version of SECISEARCH3 (Mariotti et al., 2013) that was current

173 as of 5 May 2017 with the default parameters. Assembled and annotated sequences of Hypopitys

174 monotropa mitochondrial genome are deposited in the NCBI Genbank under accession numbers

175 MK990822 and MK990823.

176 Gene Ontology (GO) terms were assigned to the genes of the mitochondrial genome by the

177 NetGO 2.0 server (You et al., 2019). Genes with their GO terms are provided in Supplementary

178 Table S2. GO terms with prediction scores $<=0.6$ are considered of low confidence in the

179 manual of NetGO and were therefore discarded.

180 2.4. RNA editing analysis

181 To obtain information on RNA editing sites, we artificially spliced gene sequences and mapped 182 RNA-seq reads to the sequences with the parameters indicated above for RNA-seq read

183 mapping. Variant calling was performed by using CLC Genomics Workbench 7.5.1. We

184 considered a site in a CDS to be edited if it was covered by at least 20 reads and at least $10 \%$ of

185 the reads in the corresponding positions differed from the genomic sequence.

186 To estimate the fraction of RNA editing sites that change the amino acid sequences of the 187 corresponding proteins, we used a measure that has been used in several previous studies (Chen, 188 2013; Xu and Zhang, 2014; Zhu et al., 2014) but has not yet been given a name. Here, we refer 189 to this measure as $\mathrm{dEN} / \mathrm{dES}$. It is calculated similarly to $\mathrm{dN} / \mathrm{dS}$, but while $\mathrm{dN} / \mathrm{dS}$ represents the 190 ratio of nonsynonymous and synonymous substitutions per nonsynonymous and synonymous 191 sites, dEN/dES ("E" stands for "editing" here) represents the ratio of nonsynonymous and 192 synonymous RNA editing events per nonsynonymous and synonymous sites. If editing sites are 193 distributed in a CDS, dEN/dES will be close to one irrespective of whether change amino acids.

194 To compare the RNA editing sites in the mitochondrial mRNAs of H. monotropa with those in 195 the mitochondrial mRNAs of other species, we used data on RNA editing in Arabidopsis 196 thaliana (Giege and Brennicke, 1999) and Oryza sativa (Notsu et al., 2002). These data are 197 incorporated in the annotations of A. thaliana and O. sativa in GenBank, with accession numbers 198 NC_037304.1 and BA000029.3, respectively.

199 2.5. Search for sequences transferred to the mitogenome 
200 To identify sequences transferred from the plastome to the mitogenome (known as "MIPTs", 201 which stands for MItochondrial Plastid Transfers), we aligned the plastid genes of Camellia 202 sinensis to the sequences of the H. monotropa mitogenome. Camellia sinensis was chosen 203 because its plastome contains a complete set of typical plastid genes and is phylogenetically 204 closest to H. monotropa (as of May 2017) among all sequenced plants with such a complete set. 205 We searched for transferred genes and not intergenic regions because we were interested in 206 transfers that may have initially been functional. The proteins of Camellia were aligned to the 207 sequences of the mitochondrial chromosomes by using TBLASTN with a maximum allowed $e$ 208 value of $10^{-3}$. tRNA and rRNA coding genes were similarly aligned to the sequences of the 209 chromosomes by using BLASTN. The matching regions of the chromosomes were then aligned 210 by BLASTX to the NCBI nr database (for regions that matched Camellia proteins) and by 211 BLASTN to the NCBI nt database (for regions that matched Camellia RNA coding genes). If the 212 best matches in the database for a region in the mitogenome were to sequences belonging to 213 plastomes, that region was considered a MIPT. To calculate the number of frameshift-inducing 214 indels and nonsense mutations in the transferred regions, these regions were considered together with their 200 bp-long flanking sequences on both ends and aligned to homologous genes from C. sinensis. The alignment was performed by using BLASTN with the default parameters. The resultant alignments were inspected by eye.

218 To search for possible horizontal gene transfers from fungi, the mitogenome sequences were split 219 into windows of $500 \mathrm{bps}$ each, with a step size of $50 \mathrm{bps}$. These windows were aligned by 220 BLASTX to the NCBI nr database and by BLASTN to the NCBI nt database. The maximum $e$ value allowed for the matches was $10^{-5}$. The regions that yielded significant matches to fungi were extracted and aligned back to the NCBI nr database to determine whether they were uniquely shared by $H$. monotropa and fungi.

225 The analysis of the origin of the coxl intron in H. monotropa was performed as follows. First, we aligned its sequence to the NCBI nt database by using BLASTN online with the default parameters, taking the best 100 matching sequences. To these sequences, we added the sequences of all coxl introns from Ericales that were not among the best 100 matches. Then, we aligned these sequences by using the MAFFT server at https://mafft.cbrc.jp/alignment/server/ (Katoh et al., 2019) with the default parameters. The unrooted phylogenetic tree of the cox 1 introns was built by using RAxML 8.2.12 (Stamatakis, 2014) with 20 starting maximum parsimony trees and the GTR+Gamma model. The required number of bootstrap pseudoreplicates was automatically determined by the extended majority-rule consensus tree criterion (the "autoMRE" option).

2342.6 Phylogenetic analysis.

235 Genes common to the mitochondrial genomes of 25 seed plants (atpl, atp4, atp8, atp9, ccmC, 236 cob, cox1, cox2, cox3, matR, nad1, nad2, nad3, nad4, nad4L, nad5, nad6, nad7, nad8, nad9) 237 were used for the phylogenetic analysis. Their sequences were concatenated and aligned with 238 MAFFT(Katoh et al., 2017). The phylogenetic analysis was performed using RaXML (raxmlGUI 239 v.1.3.1) with nucleotide sequences under the GTR+gamma substitution model with 1000 
240 bootstrap replicates. To infer possible horizontal transfers, all protein-coding genes found in the

241 H. monotropa mitogenome were aligned and analysed in the same way as the concatenated gene

242 set.

243 Results

244 3.1. Genome structure and gene content

245 Genome assembly resulted in two sequences. The GC content of both sequences is $\sim 45 \%$. One 246 of the sequences is circular with a length of $106 \mathrm{~Kb}$ (Figure 1). It does not exhibit any long 247 repeats; the mapping of mate-pair reads showed an absence of pairs with abnormal insert sizes, 248 suggesting that this sequence is not subject to recombination. The second, longer,

249 fragment(Figure 1) is $\sim 704 \mathrm{Kbp}$ long. It was assembled into a linear sequence and exhibits a 250 more complex structure. In particular, it contains several long repeats. As shown in Figure 2, 251 three pairs of long direct repeats were observed: the beginning of the chromosome and the $80-90$ $252 \mathrm{~Kb}$ region, the end of the chromosome and the $455-470 \mathrm{~Kb}$ region, the $415-420 \mathrm{~Kb}$ region and 253

254

255

256

257

258

259

260

261

262

263

264 the $495-500 \mathrm{~Kb}$ region. Long inverted repeats were also found between the $260-262 \mathrm{~kb}$ region and the $657-659 \mathrm{~kb}$ region. A peculiar feature is a gradual decrease in the coverage on both of its ends (Supplementary Figure S1). This suggests that copies of the mitogenome with different lengths of this repeat coexist in plant cells. Repeats at the ends of the genome are observed in the linear mitochondrial genomes of several fungi, animals and protists (see, e.g., the works of Janouškovec et al. (2013) and Kayal et al. (2012)) and play an important role in their replication (Nosek et al., 1998). A similar mechanism could mediate the replication of the linear mitochondrial chromosome in H. monotropa. There are some mate-pair links between the two chromosomes, which suggests that they may recombine. However, the coverage of the smaller chromosome by mate-pair inserts (Supplementary Figure S1) presents no sharp drops; therefore, it is unlikely that recombination is frequent.

265 To check for the presence of sequences transferred from fungi, we performed a BLAST search against all fungal sequences available from the NCBI. Although a BLASTX search identified several regions exhibiting similarity to hypothetical proteins from the Rhizophagus irregularis (Glomeromycota) genome, the same regions show high similarity to other plants as well (Supplementary Table S1). This indicates that they are not the result of recent HGT mediated by mycoheterotrophy but, rather, are a result of either the ancient integration of fungal sequences into the genome of the common ancestor of Hypopitys and other flowering plants or the parallel integration of mobile genetic elements (e.g., mitoviruses) in the fungal and plant genomes. At least one of these regions exhibits high similarity to an RNA-dependent RNA polymerase gene from a known plant mitovirus, supporting the latter explanation.

274

275 Regarding gene content, the circular chromosome contains only two full-length protein-coding genes - $c c m F c$ and $c o x l$ - and three exons of the nad5 gene, while two other exons are located in

276

277 a linear fragment. Summary data for the annotation are presented in Table 1. All genes are supported by RNA-seq reads (Supplementary Figure S2, Supplementary Table S2), and most

278

279 regions with high coverage of RNA-seq reads correspond to annotated genes and their flanking regions (Supplementary Figure S3). 
280 In general, the gene content of the H. monotropa mitogenome is typical of flowering plants. 281 Surprisingly, it is even larger than that of $V$. macrocarpon, a close photosynthetic relative: atp6 282 and $r p s 14$ are pseudogenized, and $s d h 3$ and $r p s 3$ are absent in $V$. macrocarpon. There is only one 283 pseudogene in H. monotropa, rps 14. Pseudogenization or loss of rps14 is very common in 284 flowering plants (e.g., (Figueroa et al., 1999)). In addition to the standard gene set, we found a 285 new ORF (ORF671) that hypothetically encodes a 671-aa protein. It shows low similarity to a 286 gene that encodes a hypothetical protein in the mitochondrial genomes of many flowering plants 287 (the closest match is the hypothetical mitochondrial protein Salmi_Mp020 from Salvia 288 miltiorrhiza). There are 4 genes encoding ribosomal proteins of the large subunit and 8 genes of 289 the small subunit. Regarding the RNA component of the ribosome, all three ribosomal RNAs 290 (26S, 18S, 5S) typical of plant mitochondrial genomes are present in H. monotropa. The set of tRNAs consists of 17 tRNAs that are typical of the mitogenomes of autotrophic plant species. trnD-GUC in H. monotropa is split into two exons. There are no tRNAs of plastome origin except for tRNA-Gly-GCC. A selenocysteine tRNA (tRNA-Sec) gene and a sequence required for selenocysteine insertion during translation (SECIS element) have been reported in the mitogenome of $V$. macrocarpon (Fajardo et al., 2014). However, SecMarker, a novel specialized tool for searching for tRNA-Sec (Santesmasses et al., 2017) that presents higher sensitivity and specificity than the tools used by Fajardo and co-workers, did not confirm the presence of a tRNA-Sec gene in the $V$. macrocarpon mitogenome. Thus, we infer that the predictions of a tRNA-Sec gene and a SECIS element in the $V$. macrocarpon mitogenome were false positives. In H. monotropa, we also did not find tRNA-Sec genes or SECIS elements. There is a region with moderate (78.8\%) similarity to the presumed tRNA-Sec of $V$. macrocarpon. It is located upstream of $c \mathrm{~cm} C$ as well as one of the two presumed tRNA-Sec sequences of $V$. macrocarpon. tRNA genes are known to exhibit highly conserved sequences; in particular, the similarity of other $V$. macrocarpon and $H$. monotropa tRNA genes is $98-100 \%$. This suggests that the presumed tRNA-Sec of $V$. macrocarpon is not a functional gene but a pseudogene of a tRNAcoding gene.

Despite being highly conserved in coding sequences, mitochondrial genes sometimes differ in their intron content. For example, cox 2 may consist of three exons (D. carota, $V$. vinifera), two exons (C. paramensis, A. thaliana), or a single exon ( $V$. macrocarpon). In many angiosperm lineages, the coxl gene contains a group I intron, presumably acquired via multiple horizontal transfer events (Cho et al., 1998; Sanchez-Puerta et al., 2011, 2008), although there is an opposing hypothesis indicating a single HGT event and multiple losses (Cusimano et al., 2008). The coxl intron is highly overrepresented in the parasitic plants that have been examined to date (Barkman et al., 2007), although the hypothesis that parasitism may serve as a mediator of horizontal intron transfer is not supported by phylogenetic analysis (Fan et al., 2016). $V$. macrocarpon lacks an intron in coxl, while in H. monotropa, an intron is present. Phylogenetic analysis indicates that the intron found in H. monotropa has the same origin as that in Pyrola secunda (Supplementary Figure S4) and was thus presumably vertically inherited from the common ancestor of Pyrola and Hypopitys. In other genes, H. monotropa exhibits the same 
320 intron content as $V$. macrocarpon. Three genes - nad1, nad2, and nad5-have trans-spliced 321 transcripts. Notably, the nad5 exons are located in different chromosomes-exons 1,2 and 3 aer 322 located in the linear chromosome and exons 4 and 5 in the circular one-suggesting a case of 323 interchromosome trans-splicing, which has been reported in other plants that have multichromosomal mitogenomes (Lloyd Evans et al., 2019; Sloan et al., 2012).

325 Detailed data on the genes and their intron-exon structure and expression are presented in

326 Supplementary Table S2.

327 3.2. Sequences transferred to the mitogenome

328 In the mitochondrial genome, we found fragments with high similarity to plastid genes, including both genes that are present in the H. monotropa plastome (matK, rps2, rps4) and genes that have been lost (rpoB, $C 1, C 2, p s b C, n d h J, B, y c f 2$ ) (Supplementary Table S3). These fragments were derived from horizontal transfers from plastids (MIPTs; presumably intracellular). Such events are frequent in plant mitochondria (for example, see the works of Alverson et al., (2011) and Grewe et al., (2014)). In rarer cases, interspecies horizontal transfer from the mitochondrial genomes of other plants harbouring inserts from the plastid genome may be a source of MIPTs, which is referred to as foreign MIPTs (Gandini and Sanchez-Puerta, 2017). MIPTs in nonphotosynthetic plants are of particular interest. If mitochondrial copies of plastid genes that were lost or pseudogenized in the plastome retain intact ORFs, the reverse switch from heterotrophy to autotrophy is potentially possible. The mitochondrial genome in this case acts as a "refugium" of plastid genes. The Castilleja paramensis mitogenome contains 55 full-length or nearly full-length plastid genes, and only approximately half of them are obvious pseudogenes (Fan et al., 2016). Currently, the only example of functional mitochondrial genes transferred from plastids is provided by the tRNA genes of plastid origin recruited for protein synthesis in mitochondria (Joyce and Grey, 1989). In H. monotropa, none of the sequences that originated from plastid protein-coding genes present intact ORFs, retaining less than $50 \%$ of the initial length and/or carrying multiple frameshift mutations in most cases (Supplementary Table S3).

\subsection{Mitochondrial gene expression and RNA editing}

To gain insights into mitochondrial gene expression and RNA editing and refine the sequence annotation, we sequenced and assembled the transcriptome of H. monotropa (Logacheva et al., 2016; Schelkunov et al., 2018). We observed the expression of all annotated protein-coding genes (hereafter, the expression level is defined as the number of mapped read pairs divided by gene length). A minimal expression level (0.1) is observed for the hypothetical protein ORF671. Cytochrome $c$ maturation factors are also expressed at low levels ( 3 of 4 genes have expression $<1)$. The genes of Complex I exhibit intermediate expression (1-6). The highest expression is observed for sdh4, cox3, atpl and atp9 (Supplementary Table S2). and position of editing sites differ greatly from species to species and from gene to gene. In $H$.

358 (Supplementary Table S4) under an editing-level threshold of 10\% and a coverage threshold of 20x. Most of these editing events are observed in all samples (Figure 3), and most of them are C- 
$360 \mathrm{U}$ events. However, we found at least one A-G editing event, which was present in all samples at 361 the level of $\sim 20 \%$ (see discussion below). The transcripts of 33 genes out of 38 are edited; the 362 maximal RNA editing density (measured as the number of sites per $100 \mathrm{bp}$ ) was observed for $363 c \mathrm{cmB}$. The genes of Complex I exhibit different RNA editing densities, ranging from 0.2 for 364 nad5 to 5.6 for nad4L. In nad4L, RNA editing restores the start codon; presumably, the same is 365 true for $V$. macrocarpon, in which $n a d 4 L$ has been (mis)annotated as a pseudogene. The RNA 366 editing density in the genes of Complexes II and III is low, not exceeding 1.1. Finally, in atpl, 367 rpl16, rps 12, 13, and ORF671, we did not find any editing events. Several genes with high 368 expression present low levels of RNA editing (atp1, rps12) and vice versa (cytochrome $c$ maturation factors), but there is generally no such tendency. The median $\mathrm{dEN} / \mathrm{dES}$ value across all genes is 1.23. There are no edited stop codons (which is expected because most of the editing events are $\mathrm{C}-\mathrm{U}$ events and stop codons lack $\mathrm{C}$; however, the observation of non-C-U editing potentially enables this), but there are several stop codons that are introduced by editing (Supplementary Table S5). To compare RNA editing in H. monotropa with that in other species, we used data from $A$. thaliana and $O$. sativa, the model plants in which RNA editing has been thoroughly characterized (Giege and Brennicke, 1999), (Notsu et al., 2002). Overall, there are 842 positions that are edited in at least one species among the protein-coding genes common to these three species (Supplementary Table S6). Only 151 (17.9\%) of these positions are edited in all three species. Notably, $37.9 \%$ of the positions are that are edited in at least one species contain a $\mathrm{T}$ in the species that do not exhibit editing in this position, while only $6.1 \%$ contain an A or G. This is congruent with the results of (Edera et al., 2018), who found that the main pattern of the loss of RNA editing is the replacement of editing sites with thymidines. A total of $337(38,1 \%)$ positions contain unedited cytosines.

\subsection{Phylogenetic analysis}

385

As mentioned above, plant mitochondrial genomes are prone to HGT. It is often detected from the incongruence of phylogenetic trees based on different genome regions (Bergthorsson et al., 2004; Cusimano and Renner, 2019). We performed phylogenetic analysis of individual mitochondrial genes and found that the topologies were similar with regard to the placement of H. monotropa - it is always placed together with $V$. macrocarpon (excluding the cases of unresolved nodes) (see Supplementary Figure S5). This evidence indicates that no genes were acquired via HGT. The combined tree of all mitochondrial genes shared across 25 seed plant species shows a topology similar to that based on nuclear and plastid genes, with monocots representing a monophyletic group and eudicots divided into two large groups - asterids and rosids. H. monotropa is sister to $V$. macrocarpon, and both species are within the asterids, as expected (Figure 4). Notably, H. monotropa genes do not exhibit any increase in substitution rates. The same is true for another MHT plant, Petrosavia stellaris (data from (Logacheva et al., 2014)). Parasitic plants were previously reported to exhibit elevated substitution rates in all three genomes (Bromham et al., 2013); however, a recent study involving broader sampling of parasitic plants shows that this is not a universal phenomenon (Zervas et al., 2019). 


\section{Discussion}

400

401

402

403

404

405

406

407

408

409

410

411

412

413

414

415

416

417

418

419

420

421

422

423

424

425

426

427

428

429

430

431

432

433

434

435

436

437

438

\subsection{Mitogenome structure}

All available evidence suggests that the $H$. monotropa mitogenome contains a linear fragment. While linear plasmids are found in the mitochondrial genomes of several plants (e.g., in the work of Handa at el., (2002)), the linear fragment of the H. monotropa mitogenome lacks characteristic features of these plasmids such as a terminal inverted repeat, small size (not over $11 \mathrm{Kbp}$ ) and genes of RNA and DNA polymerases (reviewed in (Handa, 2008)). A linear structure is typical for the mitochondrial genomes of fungi and protists (Janouškovec et al., 2013; Nosek et al., 1995). Similar to other mycoheteroprophic plants, H. monotropa lives in intimate symbiosis with fungi (Min et al., 2012). One might hypothesize that the linear fragment could be the result of either contamination or HGT from fungi. However, these explanations are unlikely to be correct for the following reasons: 1) DNA was isolated from inflorescences, while mycorrhizae exist only in roots; 2) all potential fungal hosts of $H$. monotropa with a known mitogenome possess a circular chromosome and no linear plasmids; 3 ) there are no fragments with similarity to known fungal genomes; and 4) all genes annotated in the linear fragments are typical plant mitochondrial genes. The observation that a single circular molecule ("master circle") is an oversimplified representation of the plant mitochondrial genome and that these genomes instead exist in vivo as a mixture of circular, linear and branched forms is not novel (see, for example, the report of Sloan et al. (2013)). However, a circular structure can usually be observed at the level of sequence assembly due to the presence of multiple repeats. This is not the case for H. monotropa, in which internal repeats are also present, but their location and the distribution of mate-pair links do not allow us to reconstitute the master circle (see Figure 2). This suggests that the diversity of the organization of plant mitochondrial genomes could be even greater than that reported recently (Kozik et al., 2019). An alternative explanation for the observed linear structure is that this chromosome contains a very long (longer than the size of mate-pair inserts) highly GC-rich region that cannot be sequenced using Illumina technology. Third-generation sequencing technologies, particularly the Pacific Bioscience SMRT platform, are able to handle such regions (see, e.g., Loomis et al., 2013). However, we consider this explanation to be unlikely for two reasons: first, plant mitochondrial genomes are universally biased towards AT nucleotides, and second, we would have observed GC-rich regions at the ends of the linear fragment in this case, which were not found.

A high level of convergence is observed in the gene set of plastid genomes of non-photosynthetic plants, regardless of their parasitic or mycoheterotrophic status. They are characterized by a certain degree of reduction, which usually correlates with the timing of the transition to heterotrophy (for example, see the report of Samigullin et al., (2016)) and follows the general gene loss model (Barrett et al., 2014). In contrast, the mitogenomes of heterotrophic plants are very diverse in terms of their structure, size and gene content. In H. monotropa, the total size of the mitogenome is $810 \mathrm{Kbp}$, which is almost twice as large as that of $V$. macrocarpon. However, this expansion is unlikely to be associated with heterotrophy. Large mitogenomes are known from heterotrophic plants, particularly from the MHT orchid Gastrodia elata ( $\sim 1.3 \mathrm{Mbp}$ ) (Yuan 
et al., 2018) and parasitic Cynomorium (1 Mbp) (Bellot et al., 2016) and Lophophytum mirabile (Balanophoraceae) ( $\sim 820 \mathrm{Kbp})$ (in the latter case, the size is shaped by fragments horizontally transferred from the host-see below). The other extreme is represented by the highly miniaturized mitogenomes of $V$. scurruloideum (Skippington et al., 2015).

\subsection{Horizontal gene transfer}

HGT is a very common phenomenon in parasitic plants (Yang et al., 2016). HGT from host plants into the mitochondrial genome has been shown in Rafflesiaceae (Xi et al., 2013), Orobanchaceae (Yang et al., 2016), Cynomorium (Cynomoriaceae) (Bellot et al., 2016), and Lophophytum mirabile (Balanophoraceae). In the last case, horizontally transferred homologs have replaced almost all native mitochondrial genes (Sanchez-Puerta et al., 2019, 2017). In contrast, there are no traces of HGT in the H. monotropa mitogenome. The nuclear and mitochondrial genomes of the MHT orchid Gastrodia elata were recently characterized (Yuan et al., 2018), as was the mitochondrial genome of the MHT dicot Epirixanthes elongata (Petersen et al., 2019), and no HGT was similarly observed in these cases. Although MHT plants are usually regarded alongside the parasitic plants that feed on other plants, the interactions between plants and their hosts are very different in these two cases. Parasitic plants develop specialized structures that integrate into the vascular system of a host plant and channel the flow of nutrients from the host to themselves. Such connections are similar in many aspects to graft junctions and can be the route of transport not only for nutrients but also for high-molecular-weight compounds, including proteins and nucleic acids. The bidirectional transfer of nucleic acids through haustoria in the parasitic plant Cuscuta pentagona has been shown (Kim et al., 2014). The transfer of RNA from the host is hypothesized to mediate HGT into the parasite genome. In contrast, such transfer is not known to occur in mycorrhizal symbioses. This emphasizes that despite similar heterotrophic strategies, plant parasites and MHT plants are fundamentally different in terms of the interaction with their hosts and, potentially, many other features that stem from this interaction. This calls for the increased sampling of MHT plants in genomic projects and for the development of new model systems representing MHT plants. Many of these species are rare endangered plants with very small distribution ranges; in contrast, H. monotropa is widespread and is thus presents potential as a model MHT plant.

\subsection{RNA editing}

RNA editing is an important characteristic of mitochondrial gene expression. It varies greatly in plants from complete absence in the bryophyte Marchantia polymorpha to the occurrence of several hundred or even thousands of editing events (for a review, see Ichinose and Sugita, (2016)). RNA editing in nonphotosynthetic plants is of special interest because many proteins are involved in RNA editing in both plastids and mitochondria (for example, see Bentolila et al., (2012)). Thus, the reduction of the plastid genome and the coordinated loss of nuclear genes involved in editing in plastids can also influence mitochondrial RNA editing. Currently, data on RNA editing in mitochondria for nonphotosynthetic plants are scarce. C-to-U RNA editing was found in seven genes (atp1, atp4, atp6, cox2, nad1, rps4, and rps12) in R. cantleyi (Xi et al., 
478

479

480

481

482

483

484

485

486

487

488

489

490

491

492

493

494

495

496

497

498

499

500

501

502

503

504

505

506

507

508

509

510

511

512

513

514

515

2013); and Barkman and co-authors demonstrated editing in the putatively horizontally transferred atpl gene in a wide range of plant species (Barkman et al., 2007). In $V$. scurruloideum, C-to-U editing was predicted computationally for the nine protein-coding genes (Skippington et al., 2015). In H. monotropa, we observed editing in the majority of genes; almost all editing events were of the C-to-U type, which is typical of plant mitochondria. The vast majority of the editing events were found in all samples (Figure 3, Supplementary Table S4). We observed a single non-C-U editing site, as A-G editing was observed in the rps 19 gene transcript. This editing was represented in all samples (inflorescence, ovules and anthers) at the level of 17$23 \%$ of reads (Supplementary Table S4). Considering that there was no coverage peak at this position, it is unlikely that this was a result of read mismapping. We hypothesize that the A-G conversion that we observed actually represent not A-to-G editing but adenosine-to-inosine editing (inosine reads as guanosine in sequencing data). This type of editing is typical of animals and fungi (Cattenoz et al., 2013; Liu et al., 2016) but has not previously been found in plants. In many cases, RNA editing plays a clear functional role (e.g., restoration of typical start codons in the plastid genes rpl2 and $p s b L$ (Kudla et al., 1992). We found one such case in H. monotropa (nad4L). A more detailed examination of RNA editing in MHT plants, including its dynamics in different organs and developmental stages, is required to highlight potentially functional events.

\section{Conclusions}

Nonphotosynthetic plants represent $\sim 1 \%$ of plant diversity and provide excellent models for the study of convergent evolution. Until recently, the genomic research in non-photosynthetic plants was focused on plant parasites; a common assumption is that mycoheterotrophs - plants that parasitize fungi - exhibit basically the same patterns of genome evolution as plant parasites. To test this hypothesis and to expand our knowledge of MHT plants, we characterized the mitochondrial genome of $H$. monotropa. Additionally, using RNA-seq, we performed a genomewide analysis of gene expression and RNA editing. We showed that the mitogenome structure of H. monotropa is highly unusual: it includes a small circular fragment and a large linear fragment with multiple repeats at its ends that presumably function as telomeres. Further studies that include the characterization of mitogenomes of other Ericaceae and the in vivo analysis of $H$. monotropa mitochondria are required to investigate the details of the evolution, replication and functioning of such unusual mitogenomes. The gene set is similar to that of autotrophic plants. All protein-coding genes are expressed, and in most of these genes (33 out of 38), we observed editing of the transcripts. The intergenic regions of the mitogenome carry multiple sequences of plastid origin, including sequences of photosynthesis-related genes that are absent in the $H$. monotropa plastome. We did not find any traces of HGT from fungal hosts in the H. monotropa mitogenome or an increase in nucleotide substitution rates. These new data highlight the contrast between mycoheterotrophic and parasitic plants and emphasize the need for new model species representing mycoheterotrophic plants.

\section{Funding statement}


516 This work was supported by the Russian Science Foundation (project \#17-14-01315, gene

517 expression analysis) and a budgetary subsidy to IITP RAS (project \# 0053-2019-0005, genome 518 analysis).

519 Acknowledgements

520 The authors thank Alexey Kondrashov (University of Michigan, Ann Arbor) for providing plant

521 material and Artem Kasianov for assistance with the data analysis.

522 Tables.

523 Table 1. Summary data for the structure and annotation of the H. monotropa mitogenome

524

525

526

527

528

529

530

531

532

533

534

535

536

537

538

539

540

541

542

543

544

545

546

547

548

549

550

551

552

553

554

555

556

Supplementary Table S1. Results of BLAST searches of regions showing significant similarity to fungal genes.

Supplementary Table S2. Characteristics of the mitochondrial genes of H. monotropa

Supplementary Table S3. Plastid genes in the mitogenome of H. monotropa

Supplementary Table S4. RNA editing of H. monotropa mitochondrial genes.

Supplementary Table S5.

Genes with stop codons that are introduced by editing.

\section{Supplementary Table S6.}

RNA editing of H. monotropa mitochondrial genes compared with Arabidopsis thaliana and Oryza sativa.

\section{Figures.}

Figure 1. Maps of the mitochondrial chromosomes of Hypopitys monotropa.

Trans-spliced introns are indicated by three coloured lines: red in nad1, green in nad2, and blue in nad5.

Figure 2. Repeats and mate-pair links in the mitochondrial chromosomes of Hypopitys monotropa.

(A) Repeats within and between the chromosomes. Direct repeats are connected by blue lines, and inverted repeats are connected by orange ones.

(B) "Improper" mate-pair links indicate possible chromosome rearrangements. Read pairs with reads oriented in different directions $(\longrightarrow \longleftarrow$ or $\longleftarrow \longrightarrow$ ) are coloured black, and read pairs with reads oriented in the same direction $(\rightarrow \longrightarrow$ or $\longleftarrow \leftarrow)$ are coloured green. Only the one of the two mate-pair libraries with the longer insert sizes (8279 bp on average, standard deviation 2583 bp) was used to build this diagram. A pair is considered improper if its reads are not mapped in 
557 the $\rightarrow \leftarrow$ orientation, are mapped on different chromosomes, or are mapped in the $\longrightarrow \leftarrow$

558 orientation but are separated by more than 20000 bp. Closely located reads in the $\rightarrow \longrightarrow$ and $\leftarrow$

$559 \leftarrow$ orientations that form a green "torus" across the genome $(1.8 \%$ of all mapped mate-pair

560 reads) likely represent an artefact of library preparation.

561

562

Figure 3.

563 Venn diagram representing the occurrence of RNA editing events in different RNA-seq samples.

564

565

Figure 4.

566

567

Phylogenetic tree based on the maximum likelihood analysis of the nucleotide sequences of the

568

20 -gene set. Values above nodes indicate bootstrap support. Branch lengths are proportional to the number of substitutions.

569

570

Supplementary Figure S1. Read mapping characteristics along the mitochondrial chromosomes

571 of Hypopitys monotropa.

572

(A) Average insert size between mate-pair reads spanning different genomic positions. The

573

574

575 nearly uniform insert size distribution suggests that there are no misassemblies involving large deletions or insertions. The fluctuations at the ends of the larger chromosome result from small numbers of reads mapping to the ends of the chromosome, which is linear.

576

(B) Number of mate-pair fragments covering each of the chromosome positions. The absence of

577

578

579 positions with zero coverage suggests that there are no misassemblies involving genome fragment rearrangements. The decreases at the ends of the larger chromosome result from its linearity.

580 (C) Coverage of the chromosomes by the reads of all three sequencing libraries: the paired-end 581 library and both mate-pair libraries. The coverage, though fluctuating, never reaches zero, thus 582 suggesting the absence of misassembly. Coverage at the ends of the smaller (circular) 583 chromosome abruptly decreases approximately sixfold due to difficulty of mapping reads for which part of the read maps to the end and part to the beginning of the contig. In the larger (linear) chromosome, the gradual decrease in coverage near the edges and near the $90000 \mathrm{bp}$ and 450000 bp positions are due to the varying repeat copy numbers (see discussion in the main text).

588 Supplementary Figure $\mathbf{S 2}$

589 Coverage of $H$. monotropa mitochondrial CDS by RNA-seq reads

590 Supplementary Figure S3

591 Coverage of H. monotropa mitochondrial chromosomes by RNA-seq reads

592 Supplementary Figure $\mathbf{S 4}$

593 Phylogenetic tree of the cox1 intron. Branches with bootstrap support below 70 are collapsed.

594 Supplementary Figure S5

595 Phylogenetic trees inferred from the ML analysis of single mitochondrial genes. Branches with

596

597

598

599

600 bootstrap support below 50 are collapsed.

\section{References}

Adams, K.L., Qiu, Y.-L., Stoutemyer, M., Palmer, J.D., 2002. Punctuated evolution of mitochondrial gene content: High and variable rates of mitochondrial gene loss and 
601

602

603

604

605

606

607

608

609

610

611

612

613

614

615

616

617

618

619

620

621

622

623

624

625

626

627

628

629

630

631

632

633

634

635

636

637

638

639

640

641

642

643

644

645

transfer to the nucleus during angiosperm evolution. Proc. Natl. Acad. Sci. 99, 99059912. https://doi.org/10.1073/pnas.042694899

Alverson, A.J., Rice, D.W., Dickinson, S., Barry, K., Palmer, J.D., 2011. Origins and recombination of the bacterial-sized multichromosomal mitochondrial genome of cucumber. Plant Cell 23, 2499-2513. https://doi.org/10.1105/tpc.111.087189

Alverson, A.J., Wei, X., Rice, D.W., Stern, D.B., Barry, K., Palmer, J.D., 2010. Insights into the evolution of mitochondrial genome size from complete sequences of $<\mathrm{i}>$ Citrullus lanatus $<\mathrm{i} / \mathrm{i}>$ and Cucurbita pepo (Cucurbitaceae). Mol. Biol. Evol. 27, 1436-1448. https://doi.org/10.1093/molbev/msq029

Barkman, T.J., McNeal, J.R., Lim, S.-H., Coat, G., Croom, H.B., Young, N.D., dePamphilis, C.W., 2007. Mitochondrial DNA suggests at least 11 origins of parasitism in angiosperms and reveals genomic chimerism in parasitic plants. BMC Evol. Biol. 7, 248. https://doi.org/10.1186/1471-2148-7-248

Barrett, C.F., Freudenstein, J.V., Li, J., Mayfield-Jones, D.R., Perez, L., Pires, J.C., Santos, C., 2014. Investigating the path of plastid genome degradation in an early-transitional clade of heterotrophic orchids, and implications for heterotrophic angiosperms. Mol. Biol. Evol. 31, 3095-3112. https://doi.org/10.1093/molbev/msu252

Bellot, S., Cusimano, N., Luo, S., Sun, G., Zarre, S., Gröger, A., Temsch, E., Renner, S.S., 2016. Assembled plastid and mitochondrial genomes, as well as nuclear genes, place the parasite family Cynomoriaceae in the Saxifragales. Genome Biol. Evol. 8, 2214-2230. https://doi.org/10.1093/gbe/evw147

Bentolila, S., Heller, W.P., Sun, T., Babina, A.M., Friso, G., van Wijk, K.J., Hanson, M.R., 2012. RIP1, a member of an Arabidopsis protein family, interacts with the protein RARE1 and broadly affects RNA editing. Proc. Natl. Acad. Sci. 109, E1453-E1461. https://doi.org/10.1073/pnas.1121465109

Bergthorsson, U., Richardson, A.O., Young, G.J., Goertzen, L.R., Palmer, J.D., 2004. Massive horizontal transfer of mitochondrial genes from diverse land plant donors to the basal angiosperm Amborella. Proc. Natl. Acad. Sci. 101, 17747-17752. https://doi.org/10.1073/pnas.0408336102

Bjorkman, E., 1960. Monotropa hypopitys L. - an epiparasite on tree roots. Physiol. Plant. 13, 308-327. https://doi.org/10.1111/j.1399-3054.1960.tb08034.x

Boetzer, M., Pirovano, W., 2012. Toward almost closed genomes with GapFiller. Genome Biol. 13, R56. https://doi.org/10.1186/gb-2012-13-6-r56

Bromham, L., Cowman, P.F., Lanfear, R., 2013. Parasitic plants have increased rates of molecular evolution across all three genomes. BMC Evol. Biol. 13, 126.

Camacho, C., Coulouris, G., Avagyan, V., Ma, N., Papadopoulos, J., Bealer, K., Madden, T.L., 2009. BLAST+: architecture and applications. BMC Bioinformatics 10, 421. https://doi.org/10.1186/1471-2105-10-421

Cattenoz, P.B., Taft, R.J., Westhof, E., Mattick, J.S., 2013. Transcriptome-wide identification of A > I RNA editing sites by inosine specific cleavage. RNA 19, 257-270. https://doi.org/10.1261/rna.036202.112

Chen, L., 2013. Characterization and comparison of human nuclear and cytosolic editomes. Proc. Natl. Acad. Sci. 110, E2741-E2747. https://doi.org/10.1073/pnas.1218884110

Cho, Y., Qiu, Y.L., Kuhlman, P., Palmer, J.D., 1998. Explosive invasion of plant mitochondria by a group I intron. Proc. Natl. Acad. Sci. U. S. A. 95, 14244-14249. 
646

647

648

649

650

651

652

653

654

655

656

657

658

659

660

661

662

663

664

665

666

667

668

669

670

671

672

673

674

675

676

677

678

679

680

681

682

683

684

685

686

687

688

689

690

691

Cusimano, N., Renner, S.S., 2019. Sequential horizontal gene transfers from different hosts in a widespread Eurasian parasitic plant, Cynomorium coccineum. Am. J. Bot. 106, 679-689. https://doi.org/10.1002/ajb2.1286

Cusimano, N., Zhang, L.-B., Renner, S.S., 2008. Reevaluation of the cox 1 group I intron in Araceae and angiosperms indicates a history dominated by loss rather than horizontal transfer. Mol. Biol. Evol. 25, 265-276. https://doi.org/10.1093/molbev/msm241

Edera, A.A., Gandini, C.L., Sanchez-Puerta, M.V., 2018. Towards a comprehensive picture of Cto-U RNA editing sites in angiosperm mitochondria. Plant Mol. Biol. 97, 215-231. https://doi.org/10.1007/s11103-018-0734-9

Fajardo, D., Schlautman, B., Steffan, S., Polashock, J., Vorsa, N., Zalapa, J., 2014. The American cranberry mitochondrial genome reveals the presence of selenocysteine (tRNA-Sec and SECIS) insertion machinery in land plants. Gene 536, 336-343. https://doi.org/10.1016/j.gene.2013.11.104

Fan, W., Zhu, A., Kozaczek, M., Shah, N., Pabón-Mora, N., González, F., Mower, J.P., 2016. Limited mitogenomic degradation in response to a parasitic lifestyle in Orobanchaceae. Sci. Rep. 6, 36285. https://doi.org/10.1038/srep36285

Figueroa, P., Gómez, I., Carmona, R., Holuigue, L., Araya, A., Jordana, X., 1999. The gene for mitochondrial ribosomal protein S14 has been transferred to the nucleus in Arabidopsis thaliana. Mol. Gen. Genet. MGG 262, 139-144. https://doi.org/10.1007/s004380051068

Gandini, C.L., Sanchez-Puerta, M.V., 2017. Foreign Plastid Sequences in Plant Mitochondria are Frequently Acquired Via Mitochondrion-to-Mitochondrion Horizontal Transfer. Sci. Rep. 7. https://doi.org/10.1038/srep43402

Giege, P., Brennicke, A., 1999. RNA editing in Arabidopsis mitochondria effects $441 \mathrm{C}$ to U changes in ORFs. Proc. Natl. Acad. Sci. 96, 15324-15329. https://doi.org/10.1073/pnas.96.26.15324

Graham, S.W., Lam, V.K.Y., Merckx, V.S.F.T., 2017. Plastomes on the edge: the evolutionary breakdown of mycoheterotroph plastid genomes. New Phytol. 214, 48-55. https://doi.org/10.1111/nph.14398

Grewe, F., Edger, P.P., Keren, I., Sultan, L., Pires, J.C., Ostersetzer-Biran, O., Mower, J.P., 2014. Comparative analysis of 11 Brassicales mitochondrial genomes and the mitochondrial transcriptome of Brassica oleracea. Mitochondrion 19, 135-143. https://doi.org/10.1016/j.mito.2014.05.008

Gualberto, J.M., Mileshina, D., Wallet, C., Niazi, A.K., Weber-Lotfi, F., Dietrich, A., 2014. The plant mitochondrial genome: Dynamics and maintenance. Biochimie 100, 107-120. https://doi.org/10.1016/j.biochi.2013.09.016

Handa, H., 2008. Linear plasmids in plant mitochondria: peaceful coexistences or malicious invasions? Mitochondrion 8, 15-25.

Handa, H., 2003. The complete nucleotide sequence and RNA editing content of the mitochondrial genome of rapeseed (Brassica napus L.): comparative analysis of the mitochondrial genomes of rapeseed and Arabidopsis thaliana. Nucleic Acids Res. 31, 5907-5916.

Handa, H., Itani, K., Sato, H., 2002. Structural features and expression analysis of a linear mitochondrial plasmid in rapeseed (Brassica napus L.). Mol. Genet. Genomics 267, 797805. https://doi.org/10.1007/s00438-002-0711-4

Ichinose, M., Sugita, M., 2016. RNA editing and its molecular mechanism in plant organelles. Genes 8, 5. https://doi.org/10.3390/genes8010005 
692 Janouškovec, J., Liu, S.-L., Martone, P.T., Carré, W., Leblanc, C., Collén, J., Keeling, P.J., 2013.

693

694

695

696

697

698

699

700

701

702

703

704

705

706

707

708

709

710

711

712

713

714

715

716

717

718

719

720

721

722

723

724

725

726

727

728

729

730

731

732

733

734

735
Evolution of red algal plastid genomes: ancient architectures, introns, horizontal gene transfer, and taxonomic utility of plastid markers. PLoS ONE 8, e59001. https://doi.org/10.1371/journal.pone.0059001

Joyce, P.B., Gray, M.W., 1989. Chloroplast-like transfer RNA genes expressed in wheat mitochondria. Nucleic Acids Res. 17, 5461-5476.

Katoh, K., Rozewicki, J., Yamada, K.D., 2019. MAFFT online service: multiple sequence alignment, interactive sequence choice and visualization. Brief. Bioinform. 20, 1160 1166. https://doi.org/10.1093/bib/bbx108

Katoh, K., Rozewicki, J., Yamada, K.D., 2017. MAFFT online service: multiple sequence alignment, interactive sequence choice and visualization. Brief. Bioinform. https://doi.org/10.1093/bib/bbx108

Kayal, E., Bentlage, B., Collins, A.G., Kayal, M., Pirro, S., Lavrov, D.V., 2012. Evolution of linear mitochondrial genomes in medusozoan Cnidarians. Genome Biol. Evol. 4, 1-12. https://doi.org/10.1093/gbe/evr123

Kim, G., LeBlanc, M.L., Wafula, E.K., dePamphilis, C.W., Westwood, J.H., 2014. Plant science. Genomic-scale exchange of mRNA between a parasitic plant and its hosts. Science 345, 808-811. https://doi.org/10.1126/science.1253122

Kozik, A., Rowan, B., Lavelle, D., Berke, L., Schranz, M.E., Michelmore, R.W., Christensen, A.C., 2019. The alternative reality of plant mitochondrial DNA. bioRxiv. https://doi.org/10.1101/564278

Krzywinski, M., Schein, J., Birol, I., Connors, J., Gascoyne, R., Horsman, D., Jones, S.J., Marra, M.A., 2009. Circos: An information aesthetic for comparative genomics. Genome Res. 19, 1639-1645. https://doi.org/10.1101/gr.092759.109

Kudla, J., Igloi, G.L., Metzlaff, M., Hagemann, R., Kössel, H., 1992. RNA editing in tobacco chloroplasts leads to the formation of a translatable psbL mRNA by a $\mathrm{C}$ to $\mathrm{U}$ substitution within the initiation codon. EMBO J. 11, 1099-1103.

Liu, H., Wang, Q., He, Y., Chen, L., Hao, C., Jiang, C., Li, Y., Dai, Y., Kang, Z., Xu, J.-R., 2016. Genome-wide A-to-I RNA editing in fungi independent of ADAR enzymes. Genome Res. 26, 499-509. https://doi.org/10.1101/gr.199877.115

Lloyd Evans, D., Hlongwane, T.T., Joshi, S.V., Riaño Pachón, D.M., 2019. The sugarcane mitochondrial genome: assembly, phylogenetics and transcriptomics. PeerJ 7, e7558. https://doi.org/10.7717/peerj.7558

Logacheva, M.D., Schelkunov, M.I., Nuraliev, M.S., Samigullin, T.H., Penin, A.A., 2014. The plastid genome of mycoheterotrophic monocot Petrosavia stellaris exhibits both gene losses and multiple rearrangements. Genome Biol. Evol. 6, 238-246. https://doi.org/10.1093/gbe/evu001

Logacheva, M.D., Schelkunov, M.I., Shtratnikova, V.Y., Matveeva, M.V., Penin, A.A., 2016. Comparative analysis of plastid genomes of non-photosynthetic Ericaceae and their photosynthetic relatives. Sci. Rep. 6, 30042. https://doi.org/10.1038/srep30042

Loomis, E.W., Eid, J.S., Peluso, P., Yin, J., Hickey, L., Rank, D., McCalmon, S., Hagerman, R.J., Tassone, F., Hagerman, P.J., 2013. Sequencing the unsequenceable: Expanded CGG-repeat alleles of the fragile X gene. Genome Res. 23, 121-128. https://doi.org/10.1101/gr.141705.112 
736

737

738

739

740

741

742

743

744

745

746

747

748

749

750

751

752

753

754

755

756

757

758

759

760

761

762

763

764

765

766

767

768

769

770

771

772

773

774

775

776

777

778

779

780

Mariotti, M., Lobanov, A.V., Guigo, R., Gladyshev, V.N., 2013. SECISearch3 and Seblastian: new tools for prediction of SECIS elements and selenoproteins. Nucleic Acids Res. 41, e149. https://doi.org/10.1093/nar/gkt550

Min, S., Chang-Qin, Z., Yong-Peng, M., Welti, S., Moreau, P.-A., Selosse, M.-A., 2012. Mycorrhizal features and fungal partners of four mycoheterotrophic Monotropoideae (Ericaceae) species from Yunnan, China. Symbiosis 57, 1-13. https://doi.org/10.1007/s13199-012-0180-4

Nosek, J., Dinouël, N., Kovac, L., Fukuhara, H., 1995. Linear mitochondrial DNAs from yeasts: telomeres with large tandem repetitions. Mol. Gen. Genet. MGG 247, 61-72.

Nosek, J., Tomáška, L., Fukuhara, H., Suyama, Y., Kováč, L., 1998. Linear mitochondrial genomes: 30 years down the line. Trends Genet. 14, 184-188. https://doi.org/10.1016/S0168-9525(98)01443-7

Notsu, Y., Masood, S., Nishikawa, T., Kubo, N., Akiduki, G., Nakazono, M., Hirai, A., Kadowaki, K., 2002. The complete sequence of the rice (Oryza sativa L.) mitochondrial genome: frequent DNA sequence acquisition and loss during the evolution of flowering plants. Mol. Genet. Genomics 268, 434-445. https://doi.org/10.1007/s00438-002-0767-1

Olson, A.R., 1993. Patterns of embryo and endosperm formation in Monotropa hypopitys (Monotropaceae) from North America and Sweden. Am. J. Bot. 80, 839-846. https://doi.org/10.1002/j.1537-2197.1993.tb15299.x

Olson, A.R., 1990. Observation on the floral shoots of Monotropa hypopitys (Monotropaceae). Rhodora 92, 54-56.

Petersen, G., Cuenca, A., Møller, I.M., Seberg, O., 2015. Massive gene loss in mistletoe (Viscum, Viscaceae) mitochondria. Sci. Rep. 5. https://doi.org/10.1038/srep17588

Petersen, G., Darby, H., Lam, V.K.Y., Pedersen, H.Æ., Merckx, V.S.F.T., Zervas, A., Seberg, O., Graham, S.W., 2019. Mycoheterotrophic Epirixanthes (Polygalaceae) has a typical angiosperm mitogenome but unorthodox plastid genomes. Ann. Bot. https://doi.org/10.1093/aob/mcz114

Qiu, Y., Filipenko, S.J., Darracq, A., Adams, K.L., 2014. Expression of a transferred nuclear gene in a mitochondrial genome. Curr. Plant Biol. 1, 68-72. https://doi.org/10.1016/j.cpb.2014.08.002

Rice, D.W., Alverson, A.J., Richardson, A.O., Young, G.J., Sanchez-Puerta, M.V., Munzinger, J., Barry, K., Boore, J.L., Zhang, Y., dePamphilis, C.W., Knox, E.B., Palmer, J.D., 2013. Horizontal transfer of entire genomes via mitochondrial fusion in the angiosperm Amborella. Science 342, 1468-1473. https://doi.org/10.1126/science.1246275

Samigullin, T.H., Logacheva, M.D., Penin, A.A., Vallejo-Roman, C.M., 2016. Complete plastid genome of the recent holoparasite Lathraea squamaria reveals earliest stages of plastome reduction in Orobanchaceae. PloS One 11, e0150718. https://doi.org/10.1371/journal.pone.0150718

Sanchez-Puerta, M.V., Abbona, C.C., Zhuo, S., Tepe, E.J., Bohs, L., Olmstead, R.G., Palmer, J.D., 2011. Multiple recent horizontal transfers of the coxl intron in Solanaceae and extended co-conversion of flanking exons. BMC Evol. Biol. 11. https://doi.org/10.1186/1471-2148-11-277

Sanchez-Puerta, M.V., Cho, Y., Mower, J.P., Alverson, A.J., Palmer, J.D., 2008. Frequent, phylogenetically local horizontal transfer of the cox1 group I intron in flowering plant mitochondria. Mol. Biol. Evol. 25, 1762-1777. https://doi.org/10.1093/molbev/msn129

Peer) reviewing PDF | (2019:06:38367:3:0:NEW 28 Apr 2020) 
781

782

783

784

785

786

787

788

789

790

791

792

793

794

795

796

797

798

799

800

801

802

803

804

805

806

807

808

809

810

811

812

813

814

815

816

817

818

819

820

821

822

823

824

825

826

Sanchez-Puerta, M.V., Edera, A., Gandini, C.L., Williams, A.V., Howell, K.A., Nevill, P.G., Small, I., 2019. Genome-scale transfer of mitochondrial DNA from legume hosts to the holoparasite Lophophytum mirabile (Balanophoraceae). Mol. Phylogenet. Evol. 132, 243-250. https://doi.org/10.1016/j.ympev.2018.12.006

Sanchez-Puerta, M.V., García, L.E., Wohlfeiler, J., Ceriotti, L.F., 2017. Unparalleled replacement of native mitochondrial genes by foreign homologs in a holoparasitic plant. New Phytol. 214, 376-387. https://doi.org/10.1111/nph.14361

Santesmasses, D., Mariotti, M., Guigó, R., 2017. Computational identification of the selenocysteine tRNA (tRNASec) in genomes. PLoS Comput. Biol. 13, e1005383. https://doi.org/10.1371/journal.pcbi.1005383

Schelkunov, M.I., Penin, A.A., Logacheva, M.D., 2018. RNA-seq highlights parallel and contrasting patterns in the evolution of the nuclear genome of fully mycoheterotrophic plants. BMC Genomics 19. https://doi.org/10.1186/s12864-018-4968-3

Skippington, E., Barkman, T.J., Rice, D.W., Palmer, J.D., 2017. Comparative mitogenomics indicates respiratory competence in parasitic Viscum despite loss of complex I and extreme sequence divergence, and reveals horizontal gene transfer and remarkable variation in genome size. BMC Plant Biol. 17. https://doi.org/10.1186/s12870-017-09928

Skippington, E., Barkman, T.J., Rice, D.W., Palmer, J.D., 2015. Miniaturized mitogenome of the parasitic plant Viscum scurruloideum is extremely divergent and dynamic and has lost all nad genes. Proc. Natl. Acad. Sci. 112, E3515-E3524. https://doi.org/10.1073/pnas. 1504491112

Sloan, D.B., 2013. One ring to rule them all? Genome sequencing provides new insights into the 'master circle' model of plant mitochondrial DNA structure. New Phytol. 200, 978-985. https://doi.org/10.1111/nph.12395

Sloan, D.B., Alverson, A.J., Wu, M., Palmer, J.D., Taylor, D.R., 2012. Recent acceleration of plastid sequence and structural evolution coincides with extreme mitochondrial divergence in the angiosperm genus Silene. Genome Biol. Evol. 4, 294-306.

Stamatakis, A., 2014. RAxML version 8: a tool for phylogenetic analysis and post-analysis of large phylogenies. Bioinformatics 30, 1312-1313.

https://doi.org/10.1093/bioinformatics/btu033

Xi, Z., Wang, Y., Bradley, R.K., Sugumaran, M., Marx, C.J., Rest, J.S., Davis, C.C., 2013. Massive mitochondrial gene transfer in a parasitic flowering plant clade. PLoS Genet. 9, e1003265. https://doi.org/10.1371/journal.pgen.1003265

$\mathrm{Xu}, \mathrm{G}$., Zhang, J., 2014. Human coding RNA editing is generally nonadaptive. Proc. Natl. Acad. Sci. 111, 3769-3774. https://doi.org/10.1073/pnas.1321745111

Yang, Z., Zhang, Y., Wafula, E.K., Honaas, L.A., Ralph, P.E., Jones, S., Clarke, C.R., Liu, S., Su, C., Zhang, H., Altman, N.S., Schuster, S.C., Timko, M.P., Yoder, J.I., Westwood, J.H., dePamphilis, C.W., 2016. Horizontal gene transfer is more frequent with increased heterotrophy and contributes to parasite adaptation. Proc. Natl. Acad. Sci. U. S. A. https://doi.org/10.1073/pnas.1608765113

You, R., Yao, S., Xiong, Y., Huang, X., Sun, F., Mamitsuka, H., Zhu, S., 2019. NetGO: improving large-scale protein function prediction with massive network information. Nucleic Acids Res. 47, W379-W387. https://doi.org/10.1093/nar/gkz388

Yuan, Y., Jin, X., Liu, J., Zhao, X., Zhou, J., Wang, X., Wang, D., Lai, C., Xu, W., Huang, J., Zha, L., Liu, D., Ma, X., Wang, L., Zhou, M., Jiang, Z., Meng, H., Peng, H., Liang, Y.,

PeerJ reviewing PDF | (2019:06:38367:3:0:NEW 28 Apr 2020) 
827

828

829

830

831

832

833

834

835

836
Li, R., Jiang, C., Zhao, Y., Nan, T., Jin, Y., Zhan, Z., Yang, J., Jiang, W., Huang, L., 2018. The Gastrodia elata genome provides insights into plant adaptation to heterotrophy. Nat. Commun. 9. https://doi.org/10.1038/s41467-018-03423-5

Zervas, A., Petersen, G., Seberg, O., 2019. Mitochondrial genome evolution in parasitic plants. BMC Evol. Biol. 19. https://doi.org/10.1186/s12862-019-1401-8

Zhu, Y., Luo, H., Zhang, X., Song, J., Sun, C., Ji, A., Xu, J., Chen, S., 2014. Abundant and Selective RNA-Editing Events in the Medicinal Mushroom Ganoderma lucidum. Genetics 196, 1047-1057. https://doi.org/10.1534/genetics.114.161414 
Figure 1

Maps of the mitochondrial chromosomes of Hypopitys monotropa

Trans-spliced introns are indicated by three colored lines - red in nad1, green in nad 2 and blue in nad5.

\section{Larger, linear chromosome. 704,088 bp}

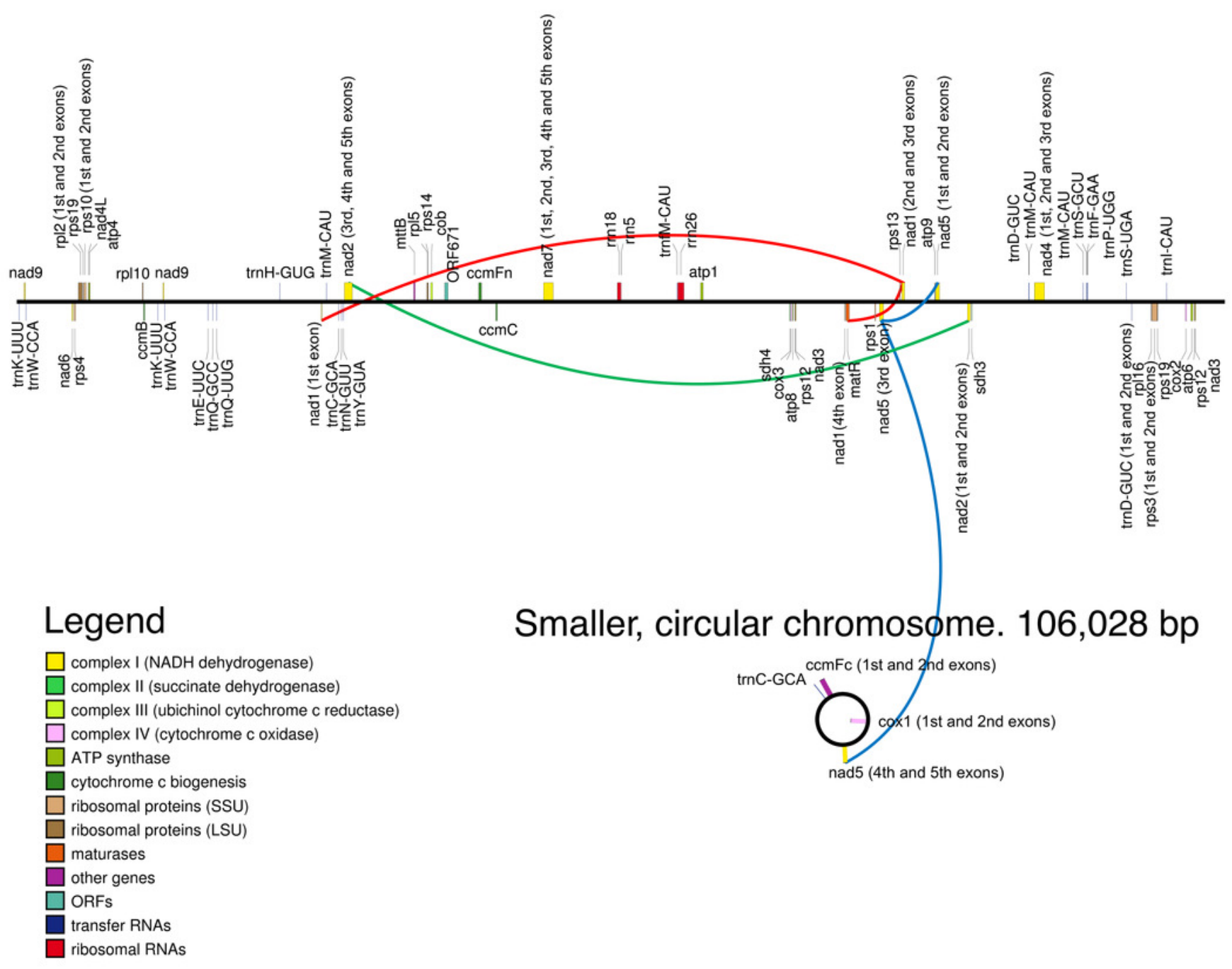




\section{Figure 2}

Repeats and mate-pair links in the mitochondrial chromosomes of Hypopitys monotropa.

(A) Repeats within and between the chromosomes. Direct repeats are connected by blue lines, inverted repeats are connected by orange ones. (B) "Improper" (with distance that exceeds expected length of the mate pair library or unexpected orientation) mate-pair links that indicate possible chromosome rearrangements. Read pairs with reads oriented in different directions $(\rightarrow \leftarrow$ or $\leftarrow \rightarrow$ ) are colored black and read pairs with reads oriented in the same direction $(\rightarrow \rightarrow$ or $\leftarrow \leftarrow$ ) are colored green. Only one of the two mate-pair libraries, that with the longer insert sizes ( 8279 bp on average, standard deviation 2583 bp), was used to build this diagram. A pair is considered improper if its reads are mapped not in the orientation $\rightarrow \leftarrow$, or are mapped on different chromosomes, or are mapped in the orientation $\rightarrow \leftarrow$ but are separated by more than $20,000 \mathrm{bp}$. The green "torus" of closely situated reads in the orientations $\rightarrow \rightarrow$ and $\leftarrow \leftarrow$ comes from mate-pair reads improperly trimmed by NextClip, the tool for mate-pair reads' trimming that we used. 

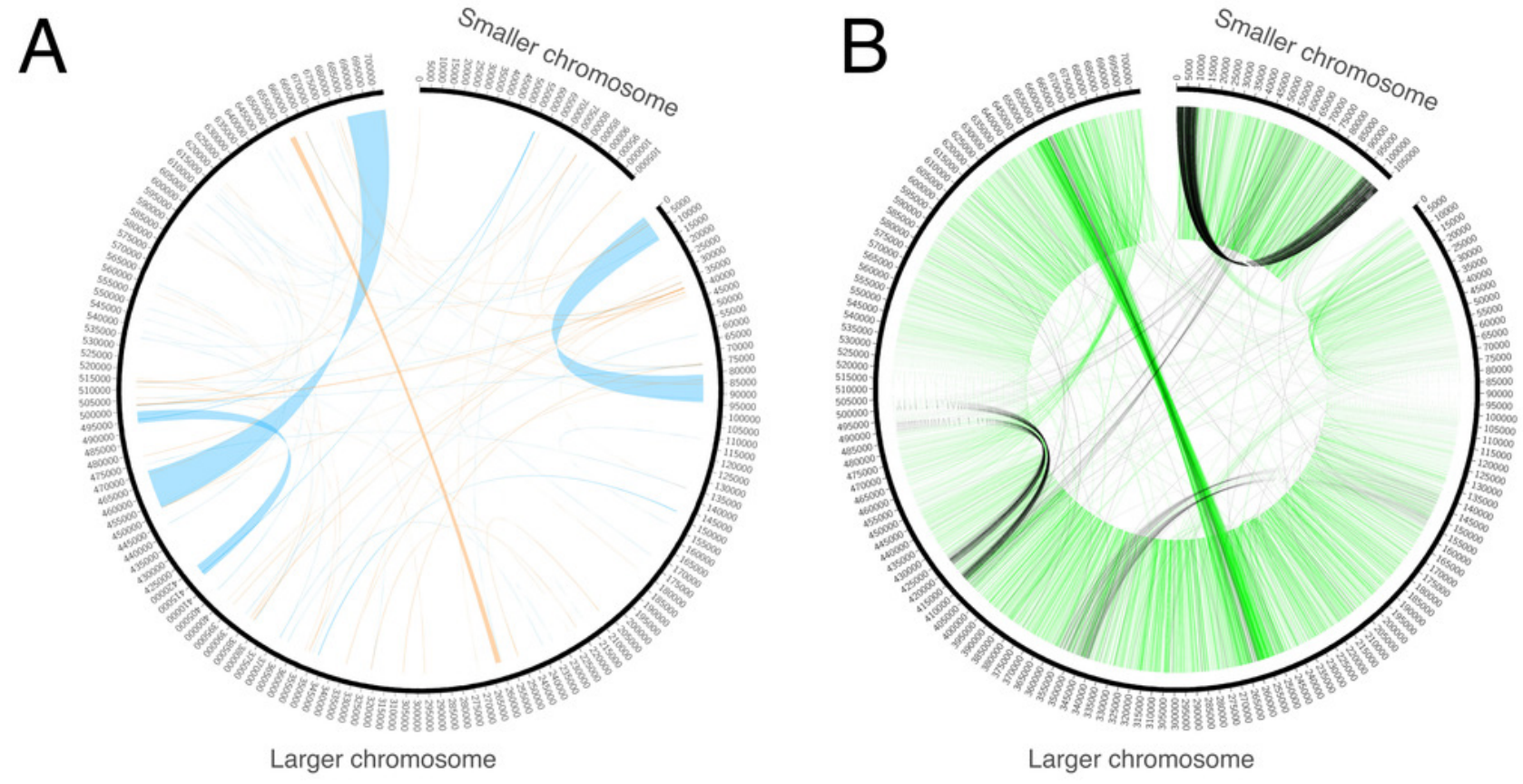
Figure 3

Venn diagram representing the occurrence of RNA editing events in different RNA-seq samples.

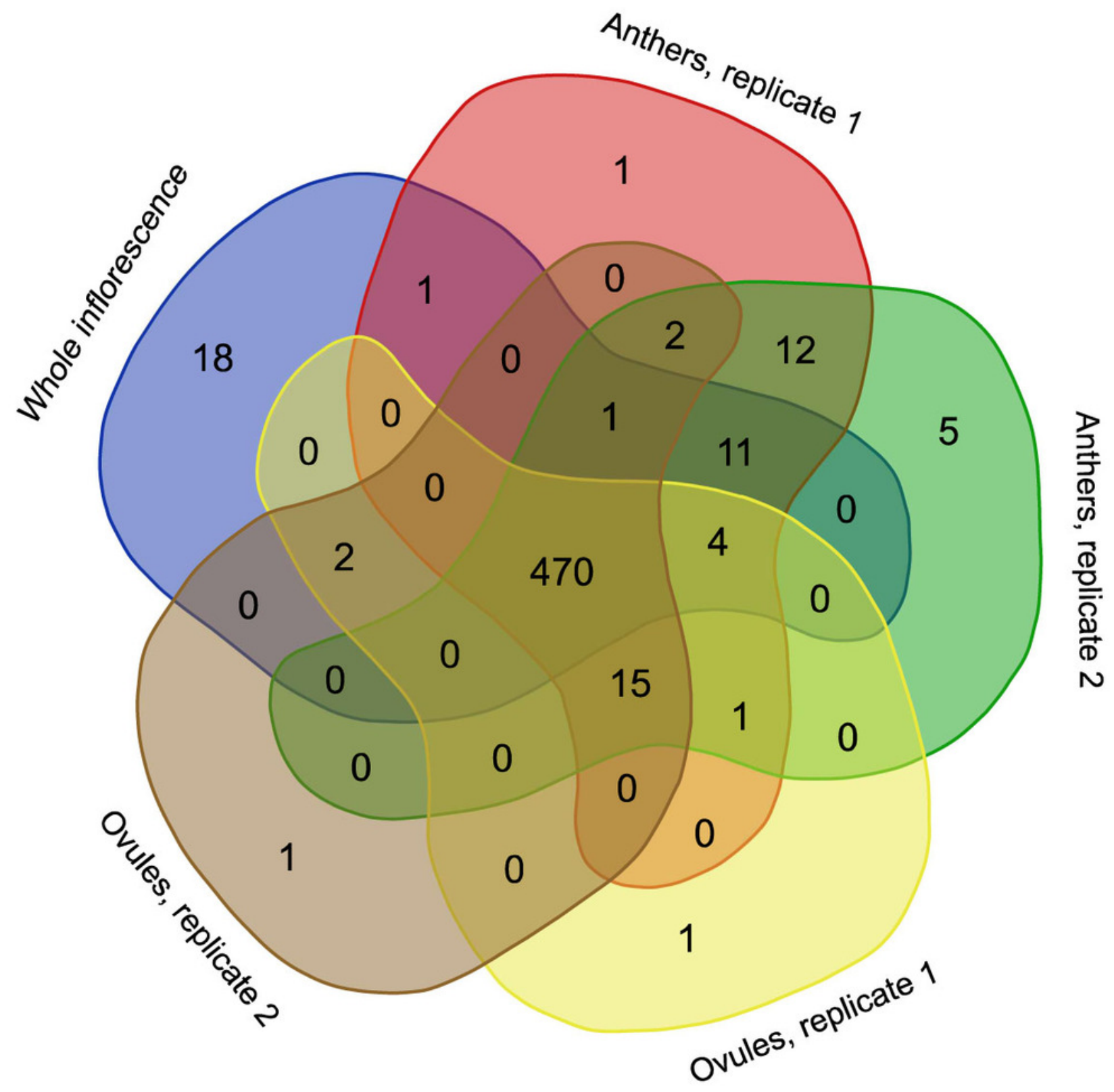


Figure 4

Phylogenetic tree based on the maximum likelihood analysis of nucleotide sequences of the 20 genes set.

Values above nodes indicate bootstrap support. Branch lengths are proportional to the number of substitutions. 


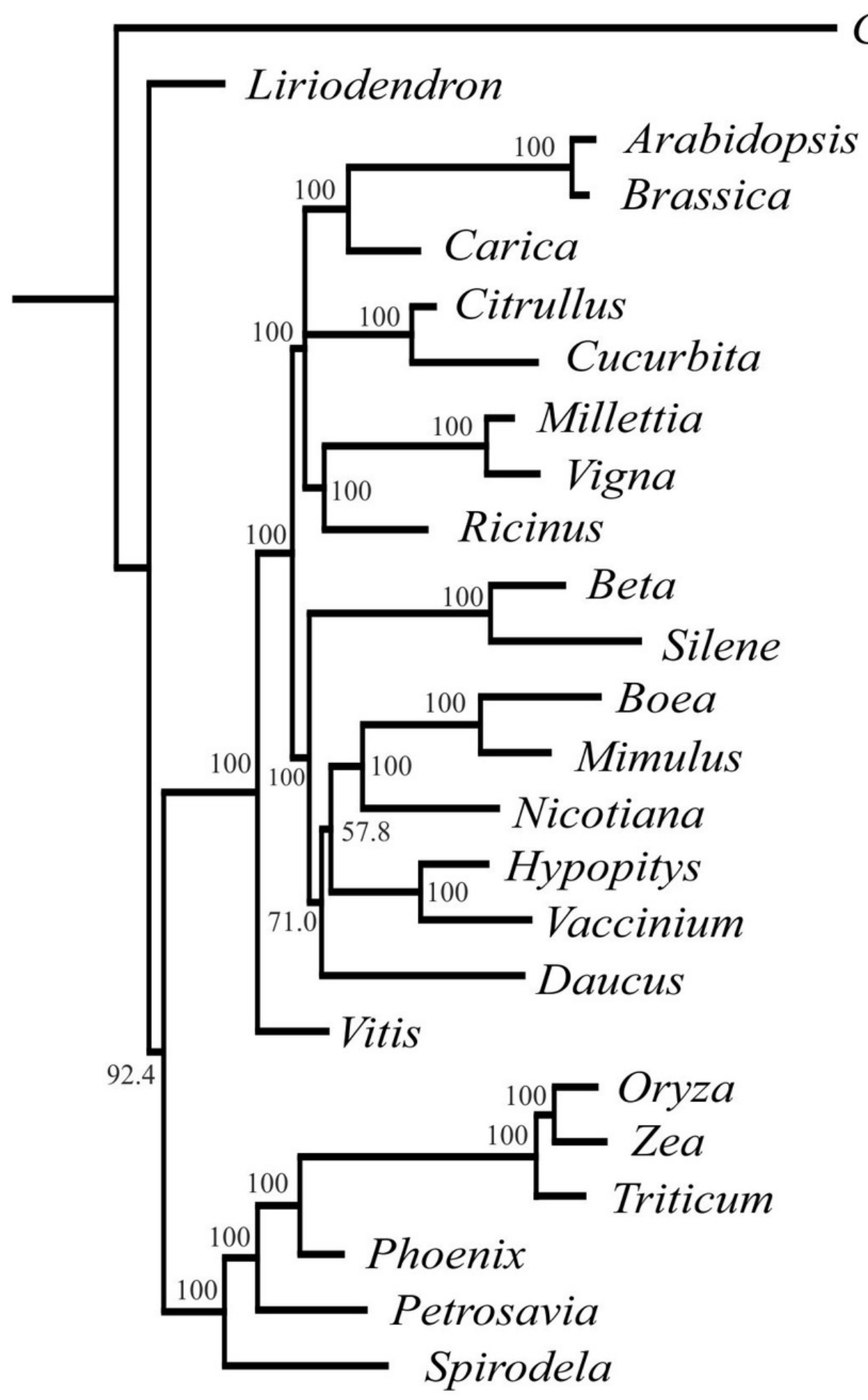

Cycas 


\section{Table 1 (on next page)}

Summary data on the structure and annotation of $H$. monotropa mitogenome. 
1 Table 1:

2 Summary data on the structure and annotation of $\boldsymbol{H}$. monotropa mitogenome.

3

\begin{tabular}{|c|c|c|c|c|c|c|c|c|c|}
\hline Chromosome & $\begin{array}{l}\text { Accessi } \\
\text { on } \\
\text { numbe } \\
\text { r } \\
\text { (NCBI) }\end{array}$ & Length & $\begin{array}{l}\text { Protein- } \\
\text { coding } \\
\text { genes }\end{array}$ & $\begin{array}{l}\text { rRNA- } \\
\text { coding } \\
\text { genes }\end{array}$ & $\begin{array}{l}\text { tRNA- } \\
\text { coding } \\
\text { genes }\end{array}$ & $\begin{array}{l}\text { Pseudo } \\
\text { genes }\end{array}$ & $\begin{array}{l}\text { Genes } \\
\text { with } \\
\text { introns }\end{array}$ & $\begin{array}{l}\text { Genes } \\
\text { with cis- } \\
\text { splicing }\end{array}$ & $\begin{array}{l}\text { Genes } \\
\text { with } \\
\text { trans- } \\
\text { splicing }\end{array}$ \\
\hline Larger (linear) & $\begin{array}{l}\text { MK990 } \\
822\end{array}$ & 704088 & 35.5 & 3 & $17-18$ & 2 & $8.5^{*}$ & 6 & 2.5 \\
\hline $\begin{array}{l}\text { Smaller } \\
\text { (circular) }\end{array}$ & $\begin{array}{l}\text { MK990 } \\
823\end{array}$ & 106028 & 2.5 & 0 & 1 & 0 & $2.5^{*}$ & 2 & 0.5 \\
\hline Total & & & 38 & 3 & $18-19$ & 1 & 11 & 8 & 3 \\
\hline
\end{tabular}

4

$5 *$ fractional number of genes indicates that one gene has exons in both circular and linear fragments. 